\title{
Attainment of reproductive competence, phase transition, and quantification of juvenility in mutant genetic screens
}

\author{
lanis G. Matsoukas ${ }^{1,2 *}$ \\ ${ }^{1}$ Engineering, Sports and Sciences Academic Group, The University of Bolton, Bolton, UK \\ ${ }^{2}$ Institute for Renewable Energy and Environmental Technologies, The University of Bolton, Bolton, UK \\ ${ }^{*}$ Correspondence: i.matsoukas@bolton.ac.uk
}

Edited by:

Christian Jung, Christian Albrechts University of Kiel, Germany

Reviewed by:

Matthias Fladung, Johann Heinrich von Thuenen Institute (vTI), Germany

Christian Jung, Christian Albrechts University of Kiel, Germany

Keywords: Arabidopsis thaliana, florigenic and antiflorigenic signaling, heteroblasty and attainment of reproductive competence, juvenile-to-adult phase transition, reciprocal transfer experiments

\section{INTRODUCTION TO JUVENILITY}

Plant development between seedling emergence and flowering is characterized by a series of successive qualitative phases: (1) a post embryonic photoperiodinsensitive phase, during which plants are insensitive to photoperiod; (2) a photoperiod-sensitive inductive phase, in which plants require a number of short day (SD) or long day (LD) inductive cycles, depending on their age for rapid flowering, and (3) a photoperiod-insensitive post-inductive phase, in which plant development is no longer influenced by photoperiod (Figure 1; Matsoukas et al., 2013).

The early phase of development during which the plants cannot be induced to flower and are effectively insensitive to environmental influences of photoperiod and/or vernalization has been called the juvenile phase (Thomas and Vince-Prue, 1997). This period differs from plant to plant from a period of a few days, for small herbaceous annual plant species, through to periods that may last longer than 20 years, as is evident for many tree species. From a physio-ecological perspective, by having a juvenile phase, plant species avoid the low seed yields that would occur if they were to flower precociously while still small and with limited photosynthetic capacity (Thomas and Vince-Prue, 1997).

\section{THE IMPORTANCE OF THE JUVENILE PHASE STUDIES}

Studies on the juvenile-to-adult phase transition have significant scientific and economic implications. Juvenility has long attracted interest as an aspect of the fundamental topic of aging and also has practical implications, especially in the growth and development of those species in which it is striking and prolonged (Matsoukas et al., 2012). From a commercial perspective, understanding the factors that affect the timing and duration of the juvenile phase length is critical for scheduling in commercial horticulture and arable crops. In addition, the long juvenile phase length of some species is one of several features limiting efficient breeding programs. For example, the efficiency of trait selection and genetic improvement in breeding programs is inversely related to the period of the breeding cycle. Thus, the exploitation of genotypes with short juvenile phase is very important. On the other hand, in many countries fast-growing tree species are being increasingly used for pulp and bioenergy production. In such cases, it may be equally important to explore molecular methods to prevent flowering and prolong juvenility (Matsoukas et al., 2012). Therefore, improving our knowledge of the ways, by which abiotic conditions and genetic factors influence juvenility and floral induction could help with crop scheduling, decrease time to flowering, and reduce waste with resulting benefits for the environment through lower inputs and energy required per unit of marketable product (Matsoukas et al., 2012, 2013).

\section{MOLECULAR GENETICS OF THE JUVENILE-TO-ADULT PHASE TRANSITION}

Molecular genetic analyses have provided insights into mechanisms that regulate the juvenile-to-adult and vegetative-toreproductive phase transitions in several plant model systems (reviewed in Jansson and Douglas, 2007; Albani and Coupland, 2010; Huijser and Schmid, 2011; Andres and Coupland, 2012; Bolouri Moghaddam and Den Ende, 2013). MicroRNA156 (miR156), an ambient temperatureresponsive $\mathrm{miR}$ (Lee et al., 2010) and strong floral inhibitor, is one of the central regulators of the juvenile-to-adult and vegetative-to-reproductive phase transitions in several species (Wu and Poethig, 2006; Chuck et al., 2007, 2011; Wang et al., 2011). Functional analysis of the hasty1 (hst1) mutant of Arabidopsis thaliana revealed the function of the contrasting transcriptional pattern of the phloemtransmitted miR156 (Lee et al., 2010) and miR172 (Lauter et al., 2005; Martin et al., 2009; Varkonyi-Gasic et al., 2010) in regulation of phase transitions (Wu and Poethig, 2006; Chuck et al., 2007; Jung et al., 2007; Mathieu et al., 2009). It has been shown that the juvenile-toadult phase transition is accompanied by a decrease in miR156/miR157 abundance and a concomitant increase in abundance of miR172, as well as the SQUAMOSA PROMOTER-BINDING PROTEIN-LIKE (SPL) transcription factors (TFs; Shikata et al., 2009; Wang et al., 2009; Jung et al., 2011; Shikata et al., 2012). Expression of 


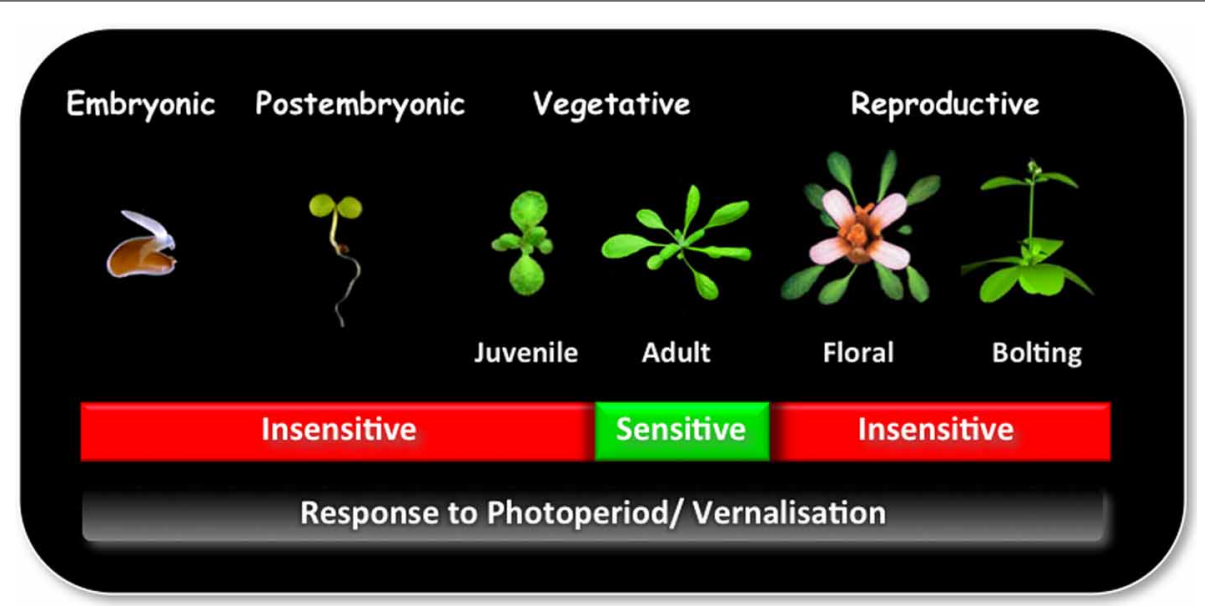

FIGURE 1 | The ability to sense and respond to photoperiod and/or vernalization varies in different phases of development. Plants undergo a series of qualitative transitions during their life-cycle in response to environmental and endogenous factors. One of the most distinguishable is the transition from a vegetative to reproductive phase of development, also known as the transition to flowering. This stage is preceded by the juvenile-to-adult phase transition within the vegetative phase. During the juvenile phase plants are incompetent to initiate reproductive development and are effectively insensitive to photoperiod and/ or vernalization. With the change to adult phase, plants attain competence to respond to floral inducers, which is required for the transition to the reproductive phase. Photoperiod is perceived in the leaf, whereas vernalization at the shoot apical meristem.
miR172 in leaves activates FLOWERING LOCUS T (FT; Aukerman and Sakai, 2003; Jung et al., 2007), the final output of the photoperiodic pathway (Corbesier et al., 2007), through repression of AP2-like transcripts SCHLAFMÜTZE (SMZ), SCHNARCHZAPFEN (SNZ) and TARGET OF EAT 1-3 (TOE1-3; Jung et al., 2007; Mathieu et al., 2009), whereas the increase in SPLs at the shoot apical meristem (SAM), leads to the transcription of floral meristem identity (FMI) genes (Wang et al., 2009; Yamaguchi et al., 2009). The FMI genes trigger the expression of floral organ identity genes (Causier et al., 2010), which function in a combinatorial fashion to specify the distinct floral organ identities.

The juvenile-to-adult phase transition is genetically regulated, although, as with most genetic traits, there are interactions with abiotic factors (Telfer and Poethig, 1998; Mohamed et al., 2010; Bergonzi et al., 2013). Arabidopsis genotypes impaired in sugar signaling, starch anabolism and catabolism, and floral repressor mutants show altered juvenile phase lengths compared to their respective wild types (Matsoukas et al., 2013). In addition, examination of diurnal metabolite changes in starch deficient and starch excess mutants indicates that their altered juvenile phase length may be due to lack of starch turnover, which influences carbohydrate availability (Matsoukas et al., 2013). Interestingly, miR156a and miR156c, the major sources of miR156 in Arabidopsis, are significantly down regulated by sugars (Yang et al., 2013; Yu et al., 2013). Furthermore, it has been shown that trehalose-6-phosphate (Tre6P) acts as a local signal that links sugar availability to the juvenile-to-adult and vegetativeto-reproductive phase transitions (Wahl et al., 2013). Arabidopsis plants impaired in Tre6P signaling pathway are late flowering. This late flowering phenotype was found to be due to reduced expression levels of FT, the elevated levels of miR156, and reduced levels of at least three miR156regulated transcripts, SPL3, SPL4, and SPL5 (Wahl et al., 2013).

\section{BIOCHEMICAL INFLUENCE}

A number of biochemical changes have been proposed to mark the juvenile-toadult phase transition in different plant species. For example, differences in peroxidase and esterase isozymes (Brand and Lineberger, 1992) and in protein phosphorylation (Huang et al., 1992). Furthermore, while various hormones have been shown to affect the juvenileto-adult phase transition, their responses sometime differ. The hormones auxin (De
Zeeuw and Leopold, 1955), abscisic acid (Rogler and Hackett, 1975), cytokinin (Mullins et al., 1979) and ethylene (Beyer and Morgan, 1971) have been demonstrated to be involved in the juvenileto-adult phase transition. In addition, gibberellic acid (GA) has promotional and repressive effects depending on plant species (Wilson et al., 1992; Chien and Sussex, 1996; Telfer et al., 1997; Telfer and Poethig, 1998). In Arabidopsis, GA mutations that affect GA biosynthesis (gal3, ga4-1, and ga5-1) and GA sensitivity (spindly4) lengthen and shorten the vegetative phase transition, respectively (Telfer et al., 1997). However, it is unclear whether alterations in various hormones levels directly control the juvenile-to-adult phase transition. The action of hormones could be indirect, for instance, by controlling partitioning or mobilization of photosynthates, and/or interacting with other hormones (Domagalska et al., 2010) and sugar signals (Zhou et al., 1998; Moore et al., 2003).

\section{MORPHOLOGICAL, HISTOLOGICAL, AND PHYSIOLOGICAL MARKERS}

In some species, the juvenile-to-adult phase transition has also been associated with several morphological, histological, and physiological traits. For instance, leaves may change in shape, size, 
phyllotaxy, and thickness. Other features associated with the developmental stage may relate to pigmentation, rooting ability, growth habit, orientation of vascular bundles, cold and disease resistance, and the physiological status of the plant (Poethig, 1990, 2003; Brunner and Nilsson, 2004; Itoh et al., 2005). However, these features are not totally reliable, since they are usually affected by different factors such as water availability, temperature, photoperiod, light quality and irradiance. In addition, the morphological, histological, and physiological changes are often less distinct in herbaceous than in woody species, and in many cases no clear association exists (Jones, 1999; Brunner and Nilsson, 2004).

In Arabidopsis the appearance of trichomes marks the juvenile-to-adult phase transition (Telfer et al., 1997). Leaves of plants in their juvenile phase of growth produce trichomes only on the adaxial surface, whereas leaves of plants in their adult phase produce trichomes on both the adaxial and abaxial surfaces. However, mutations in Arabidopsis affecting trichome development can alter trichome distribution in ways that are not phase specific (Telfer et al., 1997). This complicates the use of trichome distribution as a phase marker in mutant genetic screens.

The term "vegetative phase transition" is currently being used to characterize both heteroblasty and attainment of reproductive competence, since the two developmental events occur during the vegetative growth that precedes the transition to the reproductive phase (Poethig, 1990, 2009). However, by assessing morphological characteristics, several plant species undergo the vegetative-toreproductive phase transition while still displaying juvenile traits, and others in which floral induction does not occur, even if adult traits are appeared and the plants are treated with photo- and/or thermo-inductive conditions (Brunner and Nilsson, 2004; Poethig, 2010). This could indicate that estimation of the length of the juvenile phase based on morphological traits does not necessarily provide a reliable indication of when juvenility ends. Therefore, the use of the terms "juvenile vegetative phase" and "adult vegetative phase" in defining both the heteroblastic transition as well as the state of floral competence may lead to perplexity.

\section{COMPETENCE TO RESPOND TO FLORAL INDUCTIVE SIGNALS: A RELIABLE DETERMINANT THAT CAN BE USED TO QUANTIFY THE LENGTH OF THE JUVENILE PHASE}

The juvenile-to-adult phase transition is affected by several abiotic conditions (Matsoukas et al., 2013) and so chronological time (or the number of dormancy cycles) does not necessarily provide a reliable indication of when juvenility ends. Floral competence is the most robust determinant that can be used to distinguish between the juvenile and adult vegetative phases of plant development. However, non-flowering plants are not necessarily in their juvenile phase of development; they might be floral competent but have not been exposed to photoand/or thermo-inductive conditions for flowering.

A simple method of quantifying the length of the juvenile phase accurately and reproducibly is to conduct reciprocal transfer experiments (Mozley and Thomas, 1995; Matsoukas et al., 2013). This approach involves transferring plants at regular intervals between conditions that are inductive and non-inductive for flowering, for example between LDs and SDs (or between different levels of temperature; response to vernalization), and assess leaf number and flowering time responses (Adams et al., 2001, 2003). This approach enables the analysis of reciprocal transfer experiments data in terms of the following parameters: (1) the photoperiod-insensitive juvenile phase; (2) the photoperiod-sensitive floral inductive phases in both SDs and LDs; and (3) the photoperiod-insensitive post inductive phase (Adams et al., 2003). Plants transferred from non- or less inductive conditions to inductive conditions before the end of juvenility will exhibit similar flowering times (and for terminal flowering plant species, have the same leaf numbers), as those grown constantly in the inductive conditions (Adams et al., 2003). On the other hand, floral induction will be delayed in plants that remain under noninductive conditions after juvenility has ended. Experimental data sets obtained by the reciprocal transfer approach can be analyzed by fitting models such as those described by Adams et al. (2001, 2003). The reciprocal transfer approach has the advantage that it can be used on small seedlings, where grafting techniques are impractical, and in species where genetic analyses are not possible as little is known about the genetic regulation of the juvenile-to-adult phase transition.

\section{CONCLUDING REMARKS}

The juvenile-to-adult and vegetative-toreproductive phase transitions regulated by multiple pathways, which show different responses to external and internal stimuli. Much of the evidence for the various factors involved in the juvenile-toadult phase transition can be subject to multiple interpretations. However, it can be proposed that the prolonged juvenileto-adult and vegetative-to-reproductive phase transitions might be due to a plethora of antiflorigenic signals, which affect the transcription levels of FT and SPLs. Therefore, juvenility can be defined as the period during which the abundance of antiflorigenic signals such as miR156/miR157 is sufficiently high to suppress the expression of FT and SPL genes.

Determination of the length of the juvenile phase is a complex issue. The estimation of juvenility based on morphological, physiological, histological and biochemical markers does not necessarily provide a reliable indication of when juvenility ends. The exploitation of a single and simple experimental system to obtain accurate and reproducible estimates regarding the length of juvenility in different plant species is of crucial importance. Reproductive competence is a robust determinant that can be used to distinguish between plants that are juvenile or adult. This can be determined by conducting reciprocal transfer experiments. The simplicity of this approach enables its application in diverse plant species with comparative ease, including on young seedlings, and in genotypes where the practice of grafting is unfeasible.

\section{REFERENCES}

Adams, S. R., Munir, M., Valdes, V. M., Langton, F. A., and Jackson, S. D. (2003). Using flowering times and leaf numbers to model the phases of photoperiod sensitivity in Antirrhinum majus L. Ann. Bot. 92, 689-696. doi: 10.1093/aob/ $\operatorname{mcg} 194$ 
Adams, S. R., Pearson, S., and Hadley, P. (2001). Improving quantitative flowering models through a better understanding of the phases of photoperiod sensitivity. J. Exp. Bot. 52, 655-662. doi: 10.1093/jexbot/52.357.655

Albani, M. C., and Coupland, G. (2010). Comparative analysis of flowering in annual and perennial plants. Curr. Top. Dev. Biol. 91, 323-348. doi: 10.1016/S0070-2153(10)91011-9

Andres, F., and Coupland, G. (2012). The genetic basis of flowering responses to seasonal cues. Nat. Rev. Genet. 13, 627-639. doi: 10.1038/nrg3291

Aukerman, M. J., and Sakai, H. (2003). Regulation of flowering time and floral organ identity by a MicroRNA and its APETALA2-like target genes. Plant Cell 15, 2730-2741. doi: 10.1105/tpc.016238

Bergonzi, S., Albani, M. C., Ver Loren Van Themaat, E., Nordstrom, K. J., Wang, R., Schneeberger, K., et al. (2013). Mechanisms of age-dependent response to winter temperature in perennial flowering of Arabis alpina. Science 340, 1094-1097. doi: 10.1126/science. 1234116

Beyer, E. M., and Morgan, P. W. (1971). Abscission: the role of ethylene modification of auxin transport. Plant Physiol. 48, 208-212. doi: 10.1104/pp. 48.2.208

Bolouri Moghaddam, M. R., and Den Ende, W. V. (2013). Sugars, the clock and transition to flowering. Front. Plant Sci. 4:22. doi: 10.3389/fpls.2013. 00022

Brand, M., and Lineberger, R. (1992). In vitro rejuvenation of Betula (Betulaceae): biochemical evaluation. Am. J. Bot. 79, 626-635. doi: 10.2307/2444878

Brunner, A. M., and Nilsson, O. (2004). Revisiting tree maturation and floral initiation in the poplar functional genomics era. New Phytol. 164, 43-51. doi: 10.1111/j.1469-8137.2004.01165.x

Causier, B., Schwarz-Sommer, Z., and Davies, B. (2010). Floral organ identity: 20 years of ABCs Semin. Cell Dev. Biol. 21, 73-79. doi: 10.1016/j. semcdb.2009.10.005

Chien, J. C., and Sussex, I. M. (1996). Differential regulation of trichome formation on the adaxial and abaxial leaf surfaces by gibberellins and photoperiod in Arabidopsis thaliana (L.) Heynh. Plant Physiol. 111, 1321-1328. doi: 10.1104/pp.111.4.1321

Chuck, G. S., Cigan, A. M., Saeteurn, K., and Hake, S. (2007). The heterochronic maize mutant Corngrass1 results from overexpression of a tandem microRNA. Nat. Genet. 39, 544-549. doi: $10.1038 / \mathrm{ng} 2001$

Chuck, G. S., Tobias, C., Sun, L., Kraemer, F., Li, C. L., Dibble, D., et al. (2011). Overexpression of the maize Corngrass1 microRNA prevents flowering, improves digestibility, and increases starch content of switchgrass. Proc. Natl. Acad. Sci. U.S.A. 108, 17550-17555. doi: 10.1073/pnas.1113971108

Corbesier, L., Vincent, C., Jang, S. H., Fornara, F., Fan, Q. Z., Searle, I., et al. (2007). FT protein movement contributes to long-distance signaling in floral induction of Arabidopsis. Science 316, 1030-1033. doi: 10.1126/science. 1141752

De Zeeuw, D., and Leopold, A. C. (1955). Altering juvenility with auxin. Science 122, 925-926. doi: 10.1126/science.122.3176.925-a

Domagalska, M. A., Sarnowska, E., Nagy, F., and Davis, S. J. (2010). Genetic analyses of interactions among gibberellin, abscisic acid, and brassinosteroids in the control of flowering time in Arabidopsis thaliana. PLoS ONE 5:e14012. doi: 10.1371/journal.pone.0014012

Huang, L. C., Lius, S., Huang, B. L., Murashige, T., Mahdi El, F. M., and Van Gundy, R. (1992). Rejuvenation of Sequoia sempervirens by repeated grafting of shoot tips onto juvenile rootstocks in vitro: model for phase reversal of trees. Plant Physiol. 98, 166-173. doi: 10.1104/pp.98.1.166

Huijser, P., and Schmid, M. (2011). The control of developmental phase transitions in plants. Development 138, 4117-4129. doi: 10.1242/dev. 063511

Itoh, J., Nonomura, K., Ikeda, K., Yamaki, S., Inukai, Y., Yamagishi, H., et al. (2005). Rice plant development: from zygote to spikelet. Plant Cell Physiol. 46, 23-47. doi: 10.1093/pcp/pci501

Jansson, S., and Douglas, C. J. (2007) Populus: a model system for plant biology. Annu. Rev. Plant Biol. 58, 435-458. doi: 10.1146/annurev.arplant.58.032806.103956

Jones, C. S. (1999). An essay on juvenility, phase change, and heteroblasty in seed plants. Int. J. Plant Sci. 160, S105-S111. doi: 10.1086/314215

Jung, J. H., Seo, P. J., Kang, S. K., and Park, C. M. (2011). miR172 signals are incorporated into the miR156 signaling pathway at the SPL3/4/5 genes in Arabidopsis developmental transitions. Plant Mol. Biol. 76, 35-45. doi: 10.1007/s11103-011-9759-z

Jung, J. H., Seo, Y. H., Seo, P. J., Reyes, J. L., Yun, J., Chua, N. H., et al. (2007). The GIGANTEAregulated microRNA172 mediates photoperiodic flowering independent of CONSTANS in Arabidopsis. Plant Cell 19, 2736-2748. doi: 10.1105/tpc.107.054528

Lauter, N., Kampani, A., Carlson, S., Goebel, M., and Moose, S. P. (2005). microRNA172 down-regulates glossy15 to promote vegetative phase change in maize. Proc. Natl. Acad. Sci. U.S.A. 102, 9412-9417. doi: 10.1073/pnas.0503927102

Lee, H., Yoo, S. J., Lee, J. H., Kim, W., Yoo, S. K., Fitzgerald, H., et al. (2010). Genetic framework for flowering-time regulation by ambient temperature-responsive miRNAs in Arabidopsis. Nucleic Acids Res. 38, 3081-3093. doi: 10.1093/nar/gkp1240

Martin, A., Adam, H., Diaz-Mendoza, M., Zurczak, M., Gonzalez-Schain, N. D., and Suarez-Lopez, P. (2009). Graft-transmissible induction of potato tuberization by the microRNA miR 172 . Development 136, 2873-2881. doi: 10.1242/dev. 031658

Mathieu, J., Yant, L. J., Murdter, F., Kuttner, F., and Schmid, M. (2009). Repression of flowering by the miR172 target SMZ. PLoS Biol. 7:e1000148. doi: 10.1371/journal.pbio. 1000148

Matsoukas, I. G., Massiah, A. J., and Thomas, B. (2012). Florigenic and antiflorigenic signalling in plants. Plant Cell Physiol. 53, 1827-1842. doi: $10.1093 / \mathrm{pcp} / \mathrm{pcs} 130$

Matsoukas, I. G., Massiah, A. J., and Thomas, B. (2013). Starch metabolism and antiflorigenic signals modulate the juvenile-to-adult phase transition in Arabidopsis. Plant Cell Environ. 36, 1802-1811. doi: 10.1111/pce.12088

Mohamed, R., Wang, C. T., Ma, C., Shevchenko, O. Dye, S. J., Puzey, J. R., et al. (2010). Populus CEN/TFL1 regulates first onset of flowering, axillary meristem identity and dormancy release in
Populus. Plant J. 62, 674-688. doi: 10.1111/j.1365313X.2010.04185.x

Moore, B., Zhou, L., Rolland, F., Hall, Q., Cheng, W. H., Liu, Y. X., et al. (2003). Role of the Arabidopsis glucose sensor HXK1 in nutrient, light, and hormonal signaling. Science 300, 332-336. doi: 10.1126/science. 1080585

Mozley, D., and Thomas, B. (1995). Developmental and photobiological factors affecting photoperiodic induction in Arabidopsis thaliana Heynh. Landsberg erecta. J. Exp. Bot. 46, 173-179. doi: 10.1093/jxb/46.2.173

Mullins, M., Nair, Y., and Sampet, P. (1979). Rejuvenation in vitro: induction of juvenile characters in an adult clone of Vitis vinifera L. Ann. Bot. 44, 623-627.

Poethig, R. S. (1990). Phase change and the regulation of shoot morphogenesis in plants. Science 250, 923-930. doi: 10.1126/science.250.4983.923

Poethig, R. S. (2003). Phase change and the regulation of developmental timing in plants. Science 301, 334-336. doi: 10.1126/science.10 85328

Poethig, R. S. (2009). Small RNAs and developmental timing in plants. Curr. Opin. Genet. Dev. 19, 374-378. doi: 10.1016/j.gde.2009.06.001

Poethig, R. S. (2010). The past, present, and future of vegetative phase change. Plant Physiol. 154, 541-544. doi: 10.1104/pp.110.161620

Rogler, C., and Hackett, W. (1975). Phase change in Hedera helix: stabilization of the mature form with abscisic acid and growth retardants. Physiol. Plant. 34, 148-152. doi: 10.1111/j.13993054.1975.tb03810.x

Shikata, M., Koyama, T., Mitsuda, N., and OhmeTakagi, M. (2009). Arabidopsis SBP-box genes SPL10, SPL11 and SPL2 control morphological change in association with shoot maturation in the reproductive phase. Plant Cell Physiol. 50, 2133-2145. doi: 10.1093/pcp/pcp148

Shikata, M., Yamaguchi, H., Sasaki, K., and Ohtsubo, N. (2012). Overexpression of Arabidopsis miR157b induces bushy architecture and delayed phase transition in Torenia fournieri. Planta 236, 1027-1035. doi: 10.1007/s00425-012-1649-3

Telfer, A., Bollman, K. M., and Poethig, R. S. (1997). Phase change and the regulation of trichome distribution in Arabidopsis thaliana. Development $124,645-654$

Telfer, A., and Poethig, R. S. (1998). HASTY: a gene that regulates the timing of shoot maturation in Arabidopsis thaliana. Development 125, 1889-1898.

Thomas, B., and Vince-Prue, D. (1997). Photoperiodism in Plants. San Diego, CA: Academic Press.

Varkonyi-Gasic, E., Gould, N., Sandanayaka, M., Sutherland, P., and Macdiarmid, R. M. (2010). Characterisation of microRNAs from apple (Malus domestica "Royal Gala") vascular tissue and phloem sap. BMC Plant Biol. 10:159. doi: 10.1186/1471-2229-10-159

Wahl, V., Ponnu, J., Schlereth, A., Arrivault, S. Langenecker, T., Franke, A., et al. (2013). Regulation of flowering by trehalose-6-phosphate signaling in Arabidopsis thaliana. Science 339, 704-707. doi: 10.1126/science.1230406

Wang, J.-W., Czech, B., and Weigel, D. (2009). miR156-regulated SPL transcription factors 
define an endogenous flowering pathway in Arabidopsis thaliana. Cell 138, 738-749. doi: 10.1016/j.cell.2009.06.014

Wang, J. W., Park, M. Y., Wang, L. J., Koo, Y., Chen, X. Y., Weigel, D., et al. (2011). miRNA control of vegetative phase change in trees. PLoS Genet. 7:e1002012. doi: 10.1371/journal.pgen.10 02012

Wilson, R., Heckman, J., and Somerville, C. (1992). Gibberellin is required for flowering in Arabidopsis thaliana under short days. Plant Physiol. 100, 403-408. doi: 10.1104/pp.100.1.403

Wu, G., and Poethig, R. S. (2006). Temporal regulation of shoot development in Arabidopsis thaliana by miR156 and its target SPL3. Development 133, 3539-3547. doi: 10.1242/dev.02521

Yamaguchi, A., Wu, M. F., Yang, L., Wu, G., Poethig, R. S., and Wagner, D. (2009). The
microRNA-regulated SBP-Box transcription factor SPL3 is a direct upstream activator of LEAFY, FRUITFULL, and APETALA1. Dev. Cell 17, 268-278. doi: 10.1016/j.devcel.2009.06.007

Yang, L., Xu, M., Koo, Y., He, J., and Poethig, R. S. (2013). Sugar promotes vegetative phase change in Arabidopsis thaliana by repressing the expression of MIR156A and MIR156C. Elife 2:e00260. doi: 10.7554/eLife.00260

Yu, S., Cao, L., Zhou, C.-M., Zhang, T.-Q., Lian, H., Sun, Y., et al. (2013). Sugar is an endogenous cue for juvenile-to-adult phase transition in plants. Elife 2:e00269. doi: 10.7554/eLife.00269

Zhou, L., Jang, J. C., Jones, T. L., and Sheen, J. (1998). Glucose and ethylene signal transduction crosstalk revealed by an Arabidopsis glucoseinsensitive mutant. Proc. Natl. Acad. Sci. U.S.A. 95, 10294-10299. doi: 10.1073/pnas.95.17.10294
Received: 02 January 2014; accepted: 24 January 2014; published online: 17 February 2014.

Citation: Matsoukas IG (2014) Attainment of reproductive competence, phase transition, and quantification of juvenility in mutant genetic screens. Front. Plant Sci. 5:32. doi: 10.3389/fpls.2014.00032

This article was submitted to Plant Genetics and Genomics, a section of the journal Frontiers in Plant Science.

Copyright (c) 2014 Matsoukas. This is an open-access article distributed under the terms of the Creative Commons Attribution License (CC BY). The use, distribution or reproduction in other forums is permitted, provided the original author(s) or licensor are credited and that the original publication in this journal is cited, in accordance with accepted academic practice. No use, distribution or reproduction is permitted which does not comply with these terms. 